School of Medicine, Child Study Center, 230 South Frontage Road, New Haven, CT 06520).

COMMENT. Children and adolescents with uncomplicated Tourette syndrome (TS) are not at risk for aggressive and disruptive behavior, whereas those with comorbid TS +ADHD have a significantly higher prevalence of behavioral and conduct disorders, similar to that found with ADHD alone. Aggressive and delinquent behaviors have a negative impact on social and family functioning, adding further burdens to the quality of life of children with comorbid TS/ADHD.

\title{
PROGNOSIS OF LANGUAGE-DELAYED AND AUTISTIC PRESCHOOL CHILDREN
}

The outcome of 18 children (13 males, 5 females) with severe developmental language delay and some features of autism (not fulfilling all criteria), examined at preschool age (mean age 4 years 4 months) and again 4 years later (mean age 8 years 7 months), was evaluated at the Institute of Child Health, London, UK. At follow-up, 5 children had continuing language disorder and fulfilled diagnostic criteria for childhood autism, 4 had atypical autism with associated language disorder, and 9 had atypical autism with improved language skills. Degree of social communication impairments and repetitive behaviors at preschool evaluation correlated with severity of autism symptoms at follow-up. Parents' concerns initially were mainly about speech and language whereas at follow-up, social difficulties and social isolation were the primary concerns and inability to relate to peers. The presence of restricted, repetitive, and stereotyped behaviors in preschoolers with language delay is a negative prognostic indicator of a diagnosis of autism in later childhood. (Michelotti J, Charman T, Slonims V, Baird G. Follow-up of children with language delay and features of autism from preschool years to middle childhood. Dev Med Child Neurol Nov 2002;44:812-819). (Respond: Dr Tony Charman, Behavioural and Brain Science Unit, Institute of Child Health, 30 Guildford Street, London WC1N 1EH, UK.).

COMMENT. The relation between language delay and autism presents a diagnostic challenge in preschool children. Impairments of social interaction and repetitive behaviors reported by parents or preschool care-givers are considered risk factors for a diagnosis of autism in later childhood, especially in association with language delay. An early diagnosis of autistic spectrum disorder will permit access to appropriate interventional services.

\section{EFFECT OF LEVETIRACETAM ON MOTOR LEARNING}

The effect of the antiepileptic drug, levetiracetam, on rapid motor learning was tested in 10 healthy, right-handed volunteers (age, 22 to 43 years) by investigators from the National Institutes of Health, Bethesda, MD, and Yonsei University, Seoul, Korea. Maximum pinch force of the left thumb and index finger was determined by a pinch gauge, and the average pinch force of 5 trials was calculated before and after 30 minutes of practice at $0.5 \mathrm{~Hz}$. Either $3000 \mathrm{mg}$ of levetiracetam or placebo was administered 1 hour before the experiment. With placebo, pinch practice significantly increased pinch 
acceleration $(152 \%+/-14 \%)$, but no increase was observed after levetiracetam $(101 \%+/-$ 14\%). (Sohn YH, Jung HY, Kaelin-Lang A, Hallett M. Effect of levetiracetam on rapid motor learning in humans. Arch Neurol Dec 2002;59:1909-1912). (Respond: Mark Hallett MD, National Institute of Neurological Disorders and Stroke, National Institutes of Health, Bldg 10, Room 5N226, 10 Center Dr, MSC 1428, Bethesda, MD 20892).

COMMENT. Levetiracetam, a suppressant of motor cortex excitability, may interfere with rapid motor learning when administered to normal volunteers in a single daily dose. Previous studies (cited by the authors) have shown that this adverse effect on practice-related motor cortex plasticity is not observed with the AED, lamotrigine, although it too suppresses motor cortex excitability. These results may not apply to patients receiving long-term AED therapy.

In a clinical study of the effectiveness of levetiracetam in partial epilepsies, patients who were older at the onset of seizures did better than those with younger onset (51 vs 27 years). Also, patients with temporal lobe seizures were better controlled than those with frontal lobe localization (Bazil CW, Rose A, Resor S et al. Levetiracetam may be more effective for late-onset partial epilepsy. Arch Neurol Dec 2002;59:1905-1908).

\section{SEIZURE DISORDERS}

\section{LEARNING AND MEMORY IN CHILDREN WITH EPILEPSY}

The relation between learning and memory and epilepsy in school children with recently diagnosed idiopathic and/or cryptogenic seizures was evaluated at Wilhelmina Children's Hospital, the Netherlands. Word span (imageable nouns) and location learning of colored pictures were assessed within 48 hours after diagnosis of epilepsy and 3 and 12 months later, in 69 school children with epilepsy (aged 9.1 years, SD 2.7) and 66 classmates. Patients and controls performed similarly in registration, recall, and retention. Under conditions of increased demand on working memory (reproducing words in reverse order), patients recalled slightly less than controls; $54 \%$ of the epilepsy group, cf $26 \%$ of healthy classmates, under-performed in one or other aspects of tasks. Emotional reactions of parent and child to the onset and poor control of epilepsy contributed to impaired memory. Children with a favorable response to therapy did not differ from controls, while those with 6 months of refractory seizures had worse memory spans backwards than controls. School children with new onset idiopathic or cryptogenic epilepsy are vulnerable when processing memory tasks, particularly in tasks of increased demand and when seizures are poorly controlled. (Schouten A, Oostrom KJ, Pestman WR et al. Learning and memory of school children with epilepsy: a prospective controlled longitudinal study. Dev Med Child Neurol Nov 2002;44:803-811). (Respond: Jennekens-Schinkel A. PhD, University Medical Centre, Wilhelmina Children's Hospital, Hp KG 013271, PO Box 85090, 3508 AB Utrecht, the Netherlands).

COMMENT. School children with newly diagnosed epilepsy can retain normal learning and memory, but when task difficulty is increased or material reversed, memory may be impaired. This vulnerability to memory impairment is enhanced when patients and parents cannot adapt to the diagnosis of epilepsy or when seizures are poorly controlled. In 